\title{
HO-1 alleviates cholesterol-induced oxidative stress through activation of Nrf2/ERK and inhibition of PI3K/AKT pathways in endothelial cells
}

\author{
XIAOHAN JIN ${ }^{1,2^{*}}$, ZHONGWEI XU ${ }^{1,2^{*}}$, RONG FAN ${ }^{2}$, CHENGYAN WANG $^{2}$, WENJIE JI ${ }^{1}$, YONGQIANG MA ${ }^{1}$, \\ WEI CAI ${ }^{1}$, YAN ZHANG ${ }^{2}$, NING YANG ${ }^{1}$, SHUANG ZOU $^{2}$, XIN ZHOU $^{1}$ and YUMING LI ${ }^{1}$ \\ ${ }^{1}$ Tianjin Key Laboratory of Cardiovascular Remodeling and Target Organ Injury, \\ Pingjin Hospital Heart Center, Logistics University of People's Armed Police Force, Tianjin 300162; \\ ${ }^{2}$ Central Laboratory, Logistics University of People's Armed Police Force, Tianjin 300309, P.R. China
}

Received August 29, 2016; Accepted May 22, 2017

DOI: $10.3892 / \mathrm{mmr} .2017 .6962$

\begin{abstract}
Heme oxygenase-1 (HO-1), as an inducible and cytoprotective enzyme, has a protective effect against cellular oxidative stress. In the present study, cholesterol was used to induce lipid overload and increase reactive oxygen species (ROS), leading to oxidative stress in EA.hy926 cells. In the present study, western blotting and immunofluorescence analysis were used to detect the expression level of important molecules in the metabolism process of cholesterol. It was confirmed that cholesterol stimulation upregulated the expression of HO-1 in a time-dependent manner via the activation and translocation of nuclear factor erythroid 2-related factor 2 (Nrf2), activation of the mitogen-activated protein kinase (MAPK)/extracellular signal-regulated kinase (ERK) signaling pathway and increasing intercellular $\mathrm{Ca}^{2+}\left(\left[\mathrm{Ca}^{2+}\right]_{\mathrm{i}}\right)$ concentration. The results showed that increasing the expression of HO-1 decreased activation of the phosphoinositide 3-kinase (PI3K)/AKT signaling pathway and inhibited the expression of c-Myc. It was confirmed that cholesterol-mediated oxidative damage in vascular endothelial cells induced an increase in the expression of $\mathrm{HO}-1$ via the activation of
\end{abstract}

Correspondence to: Professor Yuming Li or Dr Xin Zhou, Tianjin Key Laboratory of Cardiovascular Remodeling and Target Organ Injury, Pingjin Hospital Heart Center, Logistics University of People's Armed Police Force, 220 Chenglin Road, Tianjin 300162, P.R. China

E-mail: cardiolab@live.com; cardiolab2016@outlook.com

E-mail: xzhou@live.com

*Contributed equally

Abbreviations: HO-1, heme oxygenase-1; ROS, reactive oxygen species; $\Delta \Psi \mathrm{m}$, mitochondrial membrane potential; $\left[\mathrm{Ca}^{2+}\right]_{\mathrm{i}}$, intercellular $\mathrm{Ca}^{2+}$

Key words: heme oxygenase-1, reactive oxygen species, cholesterol, oxidative stress
Nrf2 and the MAPK/ERK signaling pathway, and increasing the $\left[\mathrm{Ca}^{2+}\right]_{\mathrm{i}}$ concentration. The overexpression of HO-1 alleviated oxidative damage through inhibition of the PI3K/AKT signaling pathway and downregulation of the expression of c-Myc.

\section{Introduction}

Heme oxygenase-1 (HO-1), as an inducible and cytoprotective enzyme, has a protective effect against cellular oxidative stress. Studies have confirmed that cholesterol has an initiating role in several metabolic diseases, including obesity, diabetes and myocardial infarction $(1,2)$. In particular, the excessive intake of cholesterol results in lipid overload, promotes the formation of foam cells, and increases the production of pro-inflammatory factors, including interleukin (IL)-6, IL-1, tumor necrosis factor (TNF)- $\alpha$, reactive oxygen species (ROS) and cytokines $(2,3)$. These factors are released into the circulation and cause vascular endothelial damage.

HO-1, as an important molecule in the antioxidant defense system, degrades heme into carbon monoxide (CO), biliverdin and ferrous iron. HO-1 is involved in the inhibition of ROS production, the consumption of redundant ROS, and the induction of heme metabolism. Abundant ROS are usually produced under various pathological statuses, including irradiation, inflammatory processes, electron transport reactions and lipid peroxidation (4,5). High levels of ROS disrupt the balance between ROS and reactive nitrogen species, and further cause oxidative stress in the organism (6). Several studies have shown that ROS can induce the proliferation of pre-adipocytes and increase the size of adipocytes (7). The mass and volume of adipocytes is increased by the activation of NADPH oxidases and endoplasmic reticulum (ER) stress, further increasing the oxidative stress status, which increases the generation of ROS in adipocytes. The accumulation of ROS can increase the expression of glucose transporter 1 (GLUT1) by increasing transcription rate and mRNA stability, which leads to increased expression levels of GLUT1 and induces the uptake of glucose in cells $(8,9)$. 
HO-1 can degrade the $\mathrm{p} 38 \alpha$ mitogen-activated protein kinase (MAPK) isoform, and then alter the ratio of $\mathrm{p} 38 \alpha$ and $\mathrm{p} 38 \beta$ to induce the cytoprotective effect of the $\mathrm{HO}-1 / \mathrm{CO}$ metabolism pathway against the release of ROS from the mitochondrial and lipid peroxidation processes (10). CO can also mimic the effect of HO-1 and degrade the p38 $\alpha$ MAPK isoform, also exerting an antioxidant effect. Biliverdin can convert into bilirubin, and bilirubin is a potent antioxidant. $\mathrm{HO}-1, \mathrm{CO}$ and biliverdin also decrease the intracellular concentrations of ROS and protect cells from injuries caused by oxidative stress (11). The accumulation of low density lipoprotein (LDL) cholesterol can increase the production of ROS, and ROS can transform LDL to cytotoxic oxidized-LDL, which results in the release of numerous pro-inflammatory cytokines by macrophages or T cells (12). An increase in the expression of TNF- $\alpha$ can decrease adhesion in endothelial cells, facilitating the transmigration of neutrophils and increase in vascular permeability, and promoting the formation of intercellular gaps. IL-6 also can alter the shape of endothelial cells and disrupt the endothelial cell-cell barrier $(13,14)$. The stimulation of long-term high levels of lipids induces the release of inflammatory factors, and an increase in the production of ROS can finally result in atherosclerosis of the coronary artery. The rise of ROS caused by high lipid levels may directly interact with Kelch-like ECH associated protein 1 (Keap1) and cause the dissociation of the Keap1/nuclear factor erythroid 2-related factor 2 (Nrf2) complex in the cytoplasm, promoting the transportation of Nrf2 into the nucleus. Nrf2 can recognize specific DNA-binding elements of the HO-1 promoter, and then increase the expression level of HO-1 (11). However, the detailed mechanisms underlying the effect of HO-1 against cholesterol-induced oxidative damage in vascular endothelial cells remains to be elucidated.

The present study was performed to investigate the molecular mechanism through which HO-1 alleviates injury in endothelial cells caused by cholesterol. It was confirmed that cholesterol stimulation upregulated the expression of HO-1 in a time-dependent manner. The upregulated expression of HO-1 alleviated oxidative damage in the vascular endothelial cells by activation of the MAPK/extracellular signal-regulated kinase (ERK) pathway and inhibition of the phosphoinositide 3-kinase (PI3K)/AKT signaling pathway.

\section{Materials and methods}

Materials. H-DMEM was obtained from Basalmedia Technologies Co., Ltd. (Shanghai, China) and FBS was from Bailing Biotechnology Co., Ltd. (Lanzhou, China). Lipofectamine 3000 transfection reagent (cat. no. R0531) was from Thermo Fisher Scientific, Inc. (Waltham, MA, USA). Cholesterol was obtained from Sigma-Aldrich; Merck Millipore (Darmstadt, Germany). Tin protoporphyrin (SnPP; cat. no. sc-203452) was from Santa Cruz Biotechnology, Inc. (Dallas, TX, USA). The primary rabbit monoclonal anti-c-Myc (cat. no. ab32072), anti-ERK1+ERK2 (cat. no. ab184699), anti-phosphorylated (p-) ERK1+p-ERK2 (cat. no. ab76299), anti-nuclear factor (NF)- $\mathrm{B}$ p65 (cat. no. ab7970), anti-HO-1 (cat. no. ab52947), anti-Nrf2 (cat. no. ab62352), anti-AKT (cat. no. ab179463) and anti-p-AKT (cat. no. ab81283) antibodies were from Abcam (Cambridge, MA, USA). The cell membrane permeable calcium fluorescent probe and CellROX Orange reagent were from Yeasen Biotechnology Co., Ltd. (cat. no. 40704ES50, Shanghai, China). The mitochondrial membrane potential ( $\Delta \Psi \mathrm{m})$ assay kit with JC-1 (cat. no. C2006) was from Beyotime Institute of Biotechnology (Haimen, China). The anti-rabbit HRP-labeled secondary antibodies were from KPL, Inc. (Gaithersburg, MD, USA).

Cell culture. HEK293T and EA.hy926 cells obtained from the Shanghai Cell Resource Center of the Chinese Academy of Sciences (Shanghai, China; cat. nos. GNHu17 and GNHu39) were cultured at a $37^{\circ} \mathrm{C}$ in a humidified $5 \% \mathrm{CO}_{2}$ atmosphere in H-DMEM containing $10 \% \mathrm{FBS}$, respectively. According to a previous study (15), the EA.hy926 cells $\left(5 \times 10^{5}\right)$ were seeded into $100 \mathrm{~mm}$ plastic dishes and cultured for $24 \mathrm{~h}$, following which the cells were divided into three groups (SnPP, HO-1-overexpression and control). Cells in the SnPP group were treated with $20 \mathrm{mmol} / 1 \mathrm{SnPP}$ at $37^{\circ} \mathrm{C}$ for $24 \mathrm{~h}$; cells in the HO-1-overexpression group were transfected using TurboFect ${ }^{\mathrm{TM}}$ transfection reagent (cat. no. R0531; Thermo Fisher Scientific, Inc.), according to the manufacturer's protocol, with $10 \mu \mathrm{g}$ pCDNA3.1-HO-1 vector for $24 \mathrm{~h}$; and cells in the control group received no treatment. Following treatment, the cells in all groups were treated with $100 \mathrm{mmol} / \mathrm{l}$ cholesterol at $37^{\circ} \mathrm{C}$ for 0,12 and $24 \mathrm{~h}$. For all assays, the cells were washed in sterile PBS prior to removal of the redundant cholesterol.

MTT assay. An MTT assay was performed according to a previous study (16). Briefly, a concentration of $1 \times 10^{4}$ cells was seeded into each well of a 96-well plate (cat. no. 3599; Corning Incorporated, Corning, NY, USA). The cells in the three groups were respectively incubated with $5 \mathrm{mg} / \mathrm{ml} \mathrm{MTT}$ buffer at $37^{\circ} \mathrm{C}$ for $3 \mathrm{~h}$, following incubation with $100 \mathrm{mmol} / \mathrm{l}$ cholesterol for 0,12 and $24 \mathrm{~h}$. The optical density at $490 \mathrm{~nm}$ was measured using the SpectraMax series microplate reader (Molecular Devices, LLC, Sunnyvale, CA, USA).

Western blot analysis. EA.hy926 cells were cultured and divided into three groups as described above. Following treatment with $100 \mathrm{mmol} / 1$ cholesterol for 0,12 and $24 \mathrm{~h}$, the cells were lysed with RIPA buffer $(50 \mathrm{mmol} / \mathrm{l}$ Tris-HCl, $150 \mathrm{mmol} / \mathrm{l}$ $\mathrm{NaCl}, 24 \mathrm{mmol} / \mathrm{l}$ sodium deoxycholate, $3.68 \mathrm{mmol} / \mathrm{l} \mathrm{SDS}, 1 \%$ TritonX-100 and cocktail protease inhibitors) at $4^{\circ} \mathrm{C}$. A BCA assay was used to determine the concentration of protein, and $60 \mu \mathrm{g}$ of the protein samples were loaded and separated by $10 \%$ SDS-PAGE, followed by transfer onto a $0.22 \mu \mathrm{m}$ nitrocellulose membrane using a semi-dry electroblotter. The membranes were respectively incubated with primary antibodies $(1: 1,000)$ overnight at $4^{\circ} \mathrm{C}$, followed by incubation with secondary antibody $(1: 5,000)$ for $1 \mathrm{~h}$ at room temperature. The quantification of protein was performed using ECL immunoblotting reagent (KPL, Inc.). The gray values of bands were quantified using Scion Image software (version 4.0.3.2; Scion Corporation, Frederick, MD, USA) and were normalized with $\beta$-actin.

Analyses of $\triangle \Psi m, R O S$ and $\left[\mathrm{Ca}^{2+}\right]_{i}$. According to a previous study (17), a total of $1 \times 10^{4}$ cells were seeded into a confocal plate (cat. no. 801002; Nest Scientific, Rahway, NJ, USA). Following treatment with $100 \mathrm{mmol} / \mathrm{l}$ cholesterol for 0,12 and 

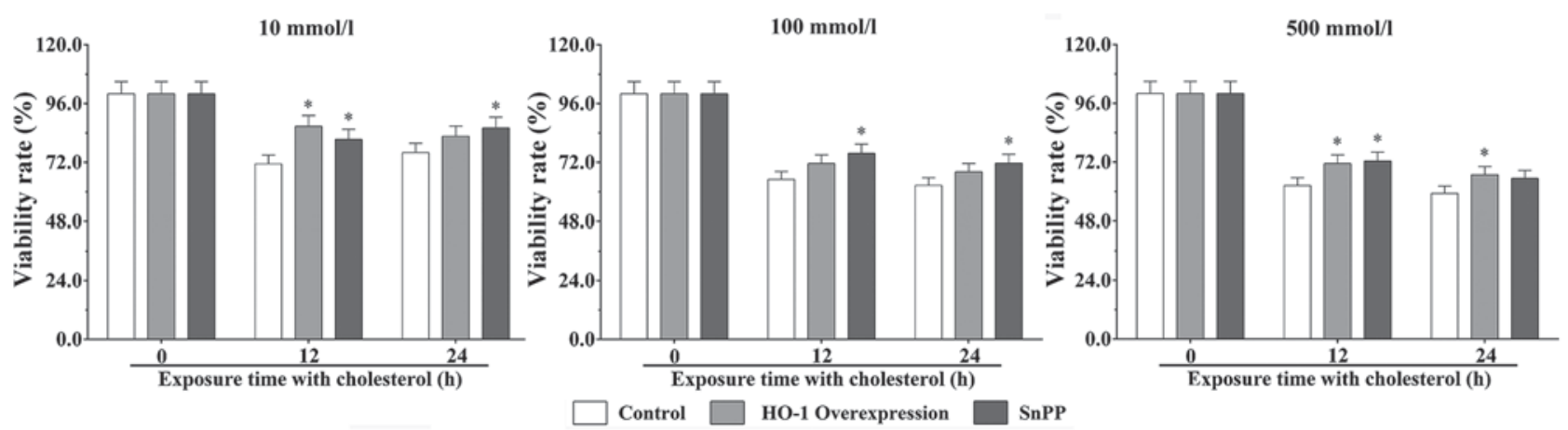

Figure 1. Effects of cholesterol on the proliferation of EA.hy926 cells. Cholesterol inhibited the proliferation of EA.hy926 cells in a time- and dose-dependent manner. Data are presented as the mean \pm standard error of the mean for each group $(n=3)$. "P<0.05, vs. control group. HO-1, heme oxygenase 1 ; SnPP, tin protoporphyrin.

$24 \mathrm{~h}$, the cells were incubated with $4 \mu \mathrm{mol} / 1$ Fluo- $4,5 \mu \mathrm{mol} / 1$ CellROX Orange reagent and $5 \mu \mathrm{g} / \mathrm{ml} \mathrm{JC}-1$ at $37^{\circ} \mathrm{C}$ for $30 \mathrm{~min}$ following removal of culture medium, respectively. Following incubation, the cells were washed with PBS three times and were then visualized using confocal microscopy (x20 objective; Leica TCS SP8; Leica Microsystems GmbH, Wetzlar, Germany). The images were analyzed using Image-Pro Plus 6.0 software (Media Cybernetics, Inc., Bethesda, MD, USA).

Statistical analysis. Data are presented as the mean \pm standard error of the mean of three independent experiments. Multi-way analysis of variance was performed to evaluate the differences between groups using GraphPad Prism 6 software (GraphPad Software Inc., La Jolla, CA, USA) followed by the Tukey-Kramer post hoc test. $\mathrm{P}<0.05$ was considered to indicate a statistically significant difference.

\section{Results}

Effects of cholesterol on the growth of EA.hy926 cells. The MTT results showed that cholesterol inhibited the proliferation and viability of EA.hy926 cells, and exhibited in a dose- and time-dependent effect (Fig. 1). Following stimulation with $10 \mathrm{mmol} / 1$ cholesterol for $12 \mathrm{~h}$, the cell viability rates in the SnPP treatment group and HO-1 overexpression group were significantly increased, compared with that in the control group $(\mathrm{P}<0.05)$. Following stimulation with cholesterol for $24 \mathrm{~h}$, the viability of the HO-1-overexpressing cells treated with $10 \mathrm{mmol} / \mathrm{l}$ cholesterol for $24 \mathrm{~h}$ was significantly increased, compared with that of the control group $(\mathrm{P}<0.05)$. Following stimulation with $100 \mathrm{mmol} / \mathrm{l}$ cholesterol for 12 and $24 \mathrm{~h}$, the viability of cells in the HO-1 overexpression group were significantly increased, compared with that of the control group $(\mathrm{P}<0.05)$. Following stimulation with $500 \mathrm{mmol} / \mathrm{l}$ cholesterol for $12 \mathrm{~h}$, the viability of cells in the SnPP treatment and HO-1 overexpression groups were significantly increased, compared with that in the control group $(\mathrm{P}<0.05)$. Following stimulation with cholesterol for $24 \mathrm{~h}$, the viability of cells in the $\mathrm{SnPP}$ treatment group was significantly increased, compared with that in the control group $(\mathrm{P}<0.05)$.

Upregulated expression of HO-1 is protective during cholesterol stimulation. The present study hypothesized that a high concentration of cholesterol stimulation results in the overexpression of HO-1 via activation of the Nrf2 signaling pathway. Under normal condition, the results of the western blot analysis showed that, compared with the control group, the expression levels of HO-1 in the cells with SnPP treatment and HO-1 overexpression were significantly increased $(\mathrm{P}<0.05)$. Following stimulation with cholesterol for $12 \mathrm{~h}$, the expression level of HO-1 was significantly increased in the HO-1 overexpression group, compared with that in the SnPP treated group $(\mathrm{P}<0.05)$. Following stimulation with cholesterol for $24 \mathrm{~h}$, the expression level of HO-1 was significantly decreased in the SnPP treated group, compared with that in the control group $(\mathrm{P}<0.05)$, and was significantly increased in the HO-1-overexpressing group, compared with that in the control group and SnPP treatment group $(\mathrm{P}<0.05$; Fig. $2 \mathrm{~A}$ and $\mathrm{B})$.

$\mathrm{Nrf} 2$ is a type of transcription activator, which can bind to antioxidant response elements (ARE) in the promoter regions of downstream target genes, including HO-1. As shown in Fig. 2C, without stimulation with cholesterol, the expression level of Nrf2 in the SnPP treatment group was significantly decreased, compared with that in the control group $(\mathrm{P}<0.05)$, and that in the HO-1 overexpression group was significantly increased, compared with that in the SnPP treatment group $(\mathrm{P}<0.05)$. Following stimulation with cholesterol for $12 \mathrm{~h}$, the expression level of Nrf2 was significantly increased in the HO-1 overexpression group, compared with levels in the control and SnPP treatment groups, respectively $(\mathrm{P}<0.05)$. Following stimulation with cholesterol for $24 \mathrm{~h}$, the expression level of Nrf2 in the HO-1 overexpression group was significantly decreased compared with the control group $(\mathrm{P}<0.05$; Fig. 2C).

$\mathrm{NF}-\mathrm{kB}$, as a pleiotropic transcription factor, can be activated by several stimuli associated with a number of biological processes, including immunity, inflammation, cell growth, differentiation, apoptosis and tumorigenesis. NF- $\kappa \mathrm{B}$ is the endpoint of several signal transduction events. As shown in Fig. 2D, without cholesterol stimulation, the expression levels of NF- $\kappa \mathrm{B}$ were significantly increased in the SnPP-treated group and HO-1 overexpression group, compared with that in the control group $(\mathrm{P}<0.05)$. Following stimulation with cholesterol for $12 \mathrm{~h}$, the expression level of NF- $\mathrm{BB}$ was significantly increased in the HO-1 overexpression group, compared with the levels in the control and SnPP-treated groups $(\mathrm{P}<0.05)$. Following stimulation with cholesterol for $24 \mathrm{~h}$, the expression level of NF-kB was significantly decreased in the SnPP-treated 
A
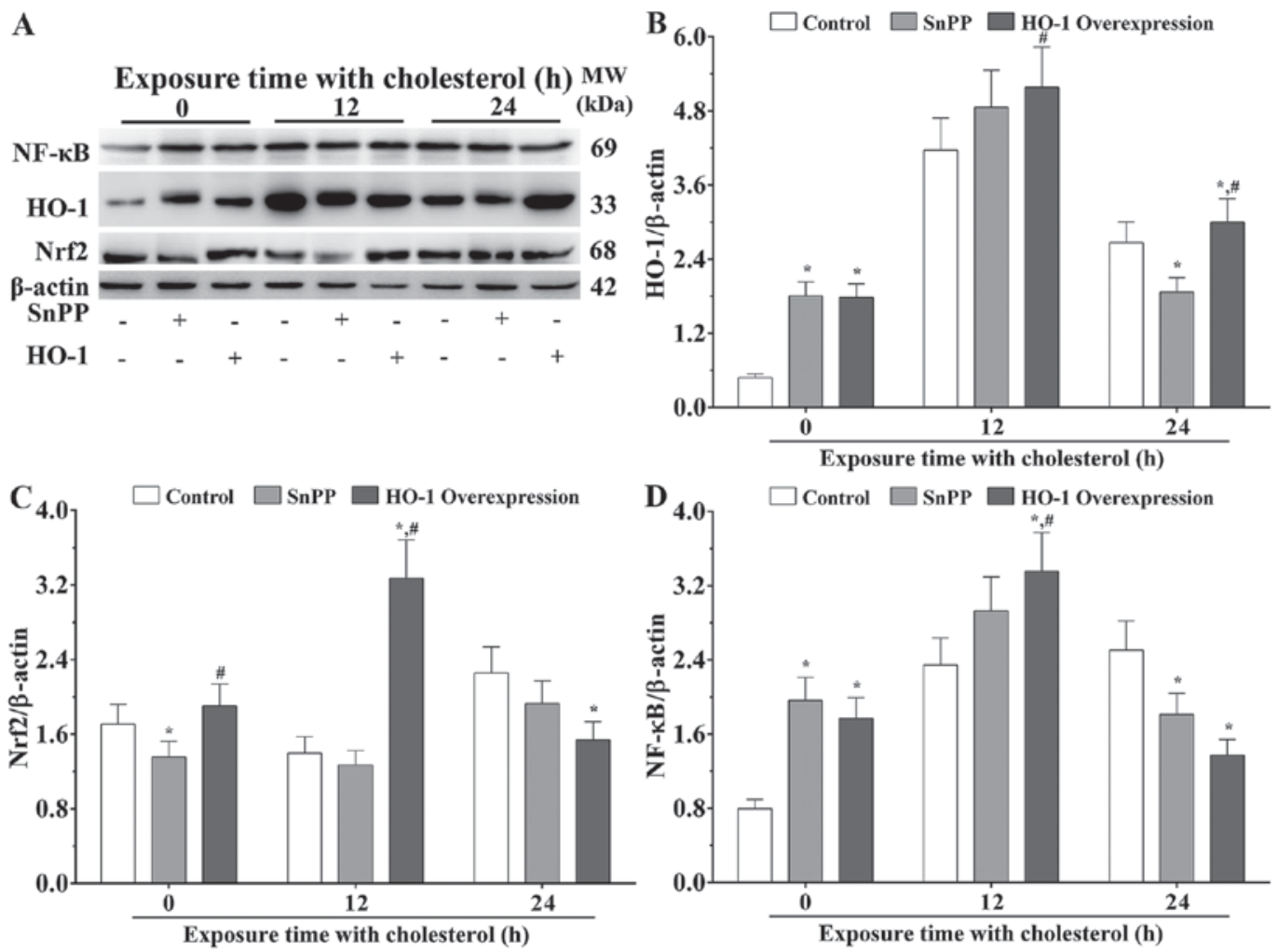

Figure 2. Effects of cholesterol on the expression of HO-1, Nrf2 and NF-kB in EA.hy926 cells stimulated with cholesterol. (A) Western blot analysis to evaluate the expression levels of HO-1, Nrf2 and NF-кB. The expression levels of (B) HO-1, (C) Nrf2 and (D) NF- $\kappa$ B were quantified by densitometry. $\beta$-actin was used as an internal control. Data are presented as the mean \pm standard error of the mean for each group $(\mathrm{n}=3)$. ${ }^{*} \mathrm{P}<0.05$, vs. control group; ${ }^{*} \mathrm{P}<0.05$, vs. SnPP group. $\mathrm{HO}-1$, heme oxygenase 1; SnPP, tin protoporphyrin; Nfr2, nuclear factor erythroid 2-related factor 2; NF- $\mathrm{kB}$, nuclear factor- $\mathrm{kB}$.

\section{A}
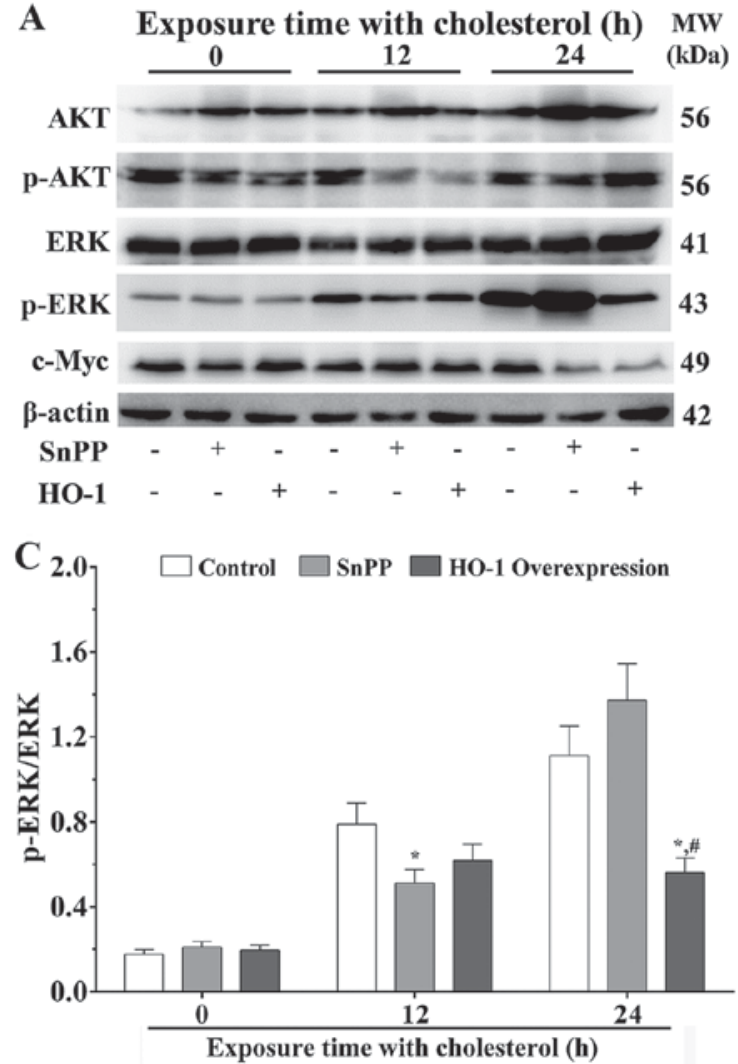
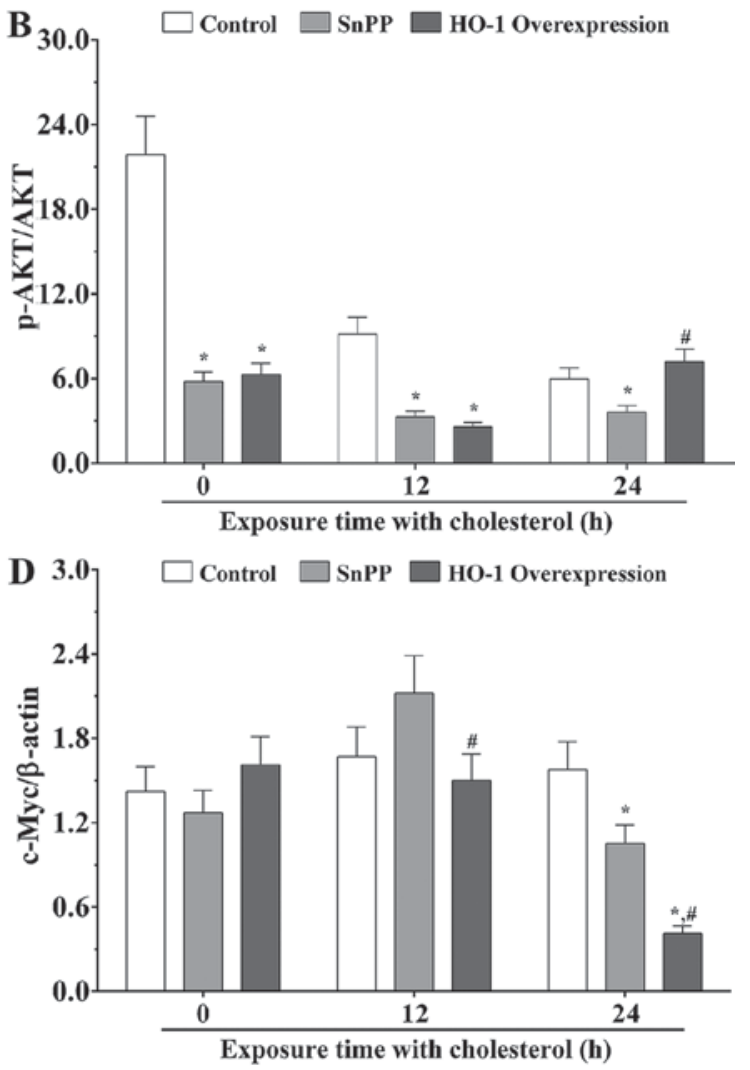

Figure 3. Effects of cholesterol on the expression of HO-1-associated molecules in EA.hy926 cells stimulated with cholesterol. (A) Western blot analysis to evaluate the expression levels of AKT, p-AKT, ERK, p-ERK and c-Myc. The expression levels of (B) AKT, p-AKT, (C) ERK, p-ERK and (D) c-Myc were quantified by densitometry. $\beta$-actin was used as an internal control. Data are presented as the mean \pm standard error of the mean for each group ( $\mathrm{n}=3$ ). ${ }^{*} \mathrm{P}<0.05$, vs. control group; ${ }^{\#} \mathrm{P}<0.05$, vs. SnPP group. HO-1, heme oxygenase 1; SnPP, tin protoporphyrin; ERK, extracellular signal-regulated kinase; p-, phosphorylated. 
group and the HO-1 overexpression group compared with the control group $(\mathrm{P}<0.05$; Fig. 2D).

Molecules involved in the regulatory progresses of $\mathrm{HO}-\mathrm{I}$ following cholesterol stimulation. The MAPK/ERK and PI3K/AKT signaling pathways are associated with the process of ROS metabolism. The results of the present study confirmed that the ratios of p-AKT/AKT were significantly decreased in the SnPP treatment group and HO-1 overexpression group, compared with that in the control group without cholesterol stimulation ( $\mathrm{P}<0.05$; Fig. $3 \mathrm{~A}$ and $\mathrm{B}$ ). Following stimulation with cholesterol for $12 \mathrm{~h}$, the ratios of $\mathrm{p}$-AKT/AKT was significantly decreased in the SnPP treatment group and HO-1 overexpression group, compared with that in the control group $(\mathrm{P}<0.05)$. Following stimulation with cholesterol for $24 \mathrm{~h}$, the ratio of $\mathrm{p}-\mathrm{AKT} / \mathrm{AKT}$ was significantly decreased in the SnPP treatment group, compared with that in the control group $(\mathrm{P}<0.05)$, and was significantly increased in the $\mathrm{HO}-1$ overexpression group, compared with that in the SnPP treatment group $(\mathrm{P}<0.05$; Fig. 3B).

The present study confirmed that there were no significant differences between the ratios of p-ERK/ERK in the three treatment groups without exposure with cholesterol. Following stimulation with cholesterol for $12 \mathrm{~h}$, the ratio of $\mathrm{p}-\mathrm{ERK} / \mathrm{ERK}$ was significantly decreased in the SnPP treatment group, compared with that in the control group $(\mathrm{P}<0.05)$. Following stimulation with cholesterol for $24 \mathrm{~h}$, the ratio of $\mathrm{p}$-ERK/ERK was significantly decreased in the $\mathrm{HO}-1$ overexpression group $(\mathrm{P}<0.05)$, compared with the ratios in the control group or SnPP treatment group (Fig. 3C).

The present study also measured the expression level of c-Myc, a downstream molecule of the MARK/ERK pathway. No significant differences were found between the expression levels of c-Myc in the three groups without cholesterol stimulation. Following stimulation with cholesterol for $12 \mathrm{~h}$, the expression of c-Myc in the HO-1 overexpression group was significantly decreased, compared with than in SnPP group $(\mathrm{P}<0.05)$. Following stimulation with cholesterol for $24 \mathrm{~h}$, the expression level of c-Myc in the SnPP treatment group was significantly decreased, compared with that in the control group $(\mathrm{P}<0.05)$, and the expression level of c-Myc in the HO-1 overexpression group was significantly decreased, compared with the ratios in the control group and SnPP treatment group $(\mathrm{P}<0.05$; Fig. 3D).

Measurements of concentrations of intracellular ROS ([ROS] $\left.{ }_{i}\right)$. Using confocal imaging, the present study measured the concentrations of $[\mathrm{ROS}]_{\mathrm{i}}$ (Fig. 4A). The mean optical intensity (MOD) values of the control group, SnPP treatment group and HO-1 overexpression group were 79.22 \pm 5.28 , $125.18 \pm 8.35$ and $80.33 \pm 5.36$, respectively, without stimulation with cholesterol. Following stimulation with cholesterol for $12 \mathrm{~h}$, the MOD values of three groups were 105.10 \pm 7.00 , $155.25 \pm 10.35$ and $119.03 \pm 7.94$. Following stimulation with

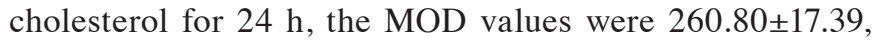
$212.02 \pm 14.13$ and $146.07 \pm 9.74$. These results indicated that, without cholesterol stimulation, $[\mathrm{ROS}]_{\mathrm{i}}$ in the SnPP treatment group was significantly increased, compared with that in the control group $(\mathrm{P}<0.05)$, whereas that of the HO-1 overexpression group was significantly decreased, compared with that of the SnPP treatment group $(\mathrm{P}<0.05)$. Following stimulation with cholesterol for $12 \mathrm{~h},[\mathrm{ROS}]_{\mathrm{i}}$ in the SnPP treatment group was significantly increased, compared with that in the control group $(\mathrm{P}<0.05)$, and $[\mathrm{ROS}] \mathrm{i}$ in the $\mathrm{HO}-1$ overexpression group was significantly decreased compared with that in the SnPP treatment group $(\mathrm{P}<0.05)$. Following stimulation with cholesterol for $24 \mathrm{~h},[\mathrm{ROS}]_{\mathrm{i}}$ in the SnPP treatment group was significantly decreased, compared with that in the control group $(\mathrm{P}<0.05)$, and $[\mathrm{ROS}] \mathrm{i}$ in the $\mathrm{HO}-1$ overexpression group was significantly decreased $(\mathrm{P}<0.05)$, compared with that in the SnPP treatment group. The quantification results of mean fluorescence intensity of $[\mathrm{ROS}]_{\mathrm{i}}$ are presented in Fig. 4B.

Measurements of $\left[\mathrm{Ca}^{2+}\right]_{i}$. The $\left[\mathrm{Ca}^{2+}\right]_{\mathrm{i}}$ was measured using confocal images, the results are shown in Fig. 4C. The MOD of the control, SnPP treatment and HO-1 overexpression groups were $127.62 \pm 6.38,2201.20 \pm 110.06$ and $98.50 \pm 4.93$, respectively, in the absence of cholesterol stimulation. Following stimulation with cholesterol for $12 \mathrm{~h}$, the MOD values of these three groups were $592.91 \pm 29.65,2,645.78 \pm 132.29$ and $212.36 \pm 10.62$. Following stimulated with cholesterol for $24 \mathrm{~h}$, the MOD values were 1,157.45 $\pm 57.87,2,831.36 \pm 141.57$ and 1.062.75 \pm 53.14 . Without stimulation with cholesterol, $\left[\mathrm{Ca}^{2+}\right]_{\mathrm{i}}$ in the SnPP treatment group was significantly increased, compared with that in the control group $(\mathrm{P}<0.05)$, and $\left[\mathrm{Ca}^{2+}\right]_{\mathrm{i}}$ in the HO-1 overexpression group was significantly decreased, compared with that in the control and SnPP treatment groups $(\mathrm{P}<0.05)$. Following stimulation with cholesterol for $12 \mathrm{~h}$, the $\left[\mathrm{Ca}^{2+}\right]_{\mathrm{i}}$ in the SnPP treatment group was significantly increased, compared with that in the control group $(\mathrm{P}<0.05)$, and $\left[\mathrm{Ca}^{2+}\right]_{\mathrm{i}}$ in the HO-1 overexpression group was significantly decreased, compared with that in the control or SnPP treatment group $(\mathrm{P}<0.05)$. Following stimulation with cholesterol for $24 \mathrm{~h},\left[\mathrm{Ca}^{2+}\right]_{\mathrm{i}}$ in the SnPP treatment group was significantly increased, compared with that in control group $(\mathrm{P}<0.05)$, whereas the level of $\left[\mathrm{Ca}^{2+}\right]_{\mathrm{i}}$ in the HO-1 overexpression group was significantly decreased, compared with that in the SnPP treatment group $(\mathrm{P}<0.05)$. The quantification results of mean fluorescence intensity of $\left[\mathrm{Ca}^{2+}\right]_{i}$ are presented in in Fig. 4D.

Measurements of $\Delta \Psi m$. The present measured the $\Delta \Psi \mathrm{m}$ in three groups using confocal imaging (Fig. 4E). The ratios of red fluorescence (JC-1 polymer)/green fluorescence (JC-1 monomer) in the three groups are shown in Fig. 4F. Without cholesterol stimulation and with cholesterol stimulation for $12 \mathrm{~h}$, the ratio of JC-1 polymer/JC-1 monomer in the SnPP treatment group was significantly decreased $(\mathrm{P}<0.05)$, compared with the ratios in the control or HO-1 overexpression group. Following stimulation with cholesterol for $24 \mathrm{~h}$, the ratio in the SnPP treatment group was significantly decreased, compared with that in the control group $(\mathrm{P}<0.05)$, and that in the $\mathrm{HO}-1$ overexpression group was significantly increased, compared with the ratios in the control and SnPP treatment groups $(\mathrm{P}<0.05)$.

\section{Discussion}

HO-1, an essential enzyme in heme catabolism, is activated in conditions of high concentrations of heme and other pathophysiological statuses, including oxidative stress, high 
A

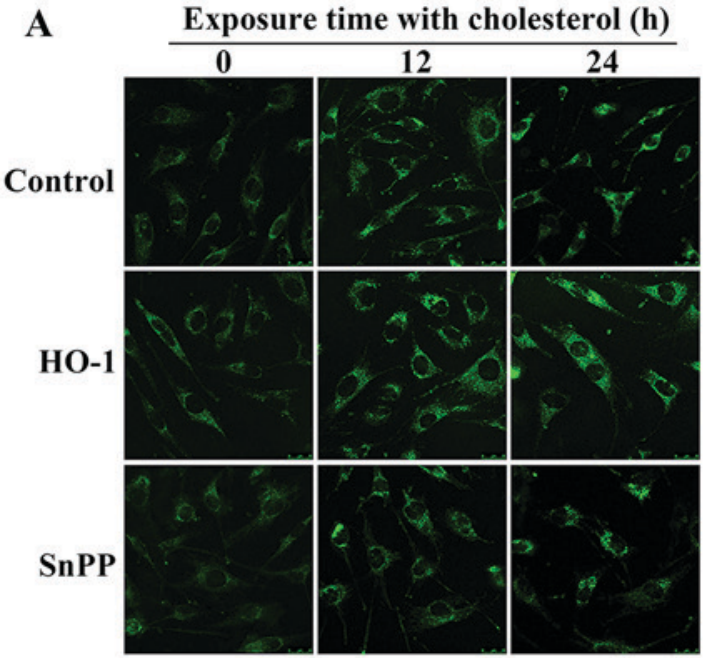

C
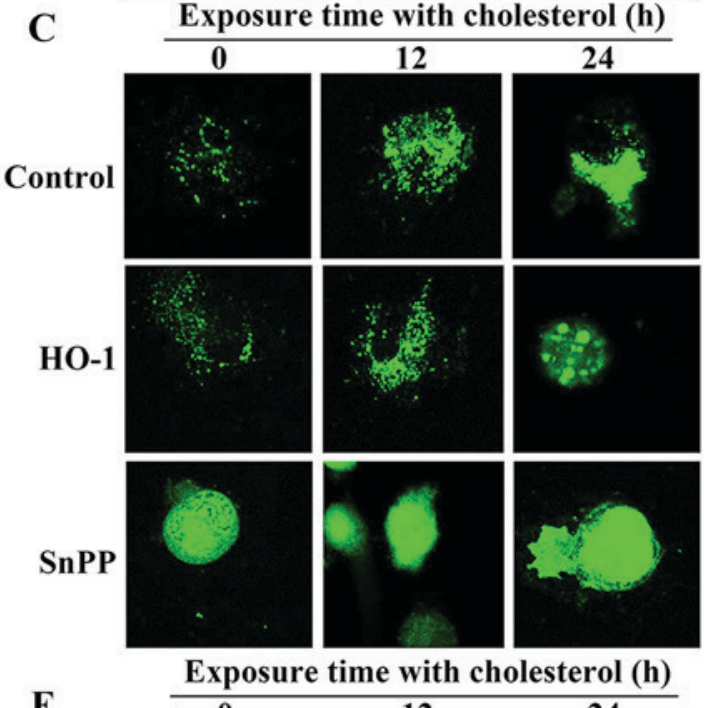

$\mathbf{E}$
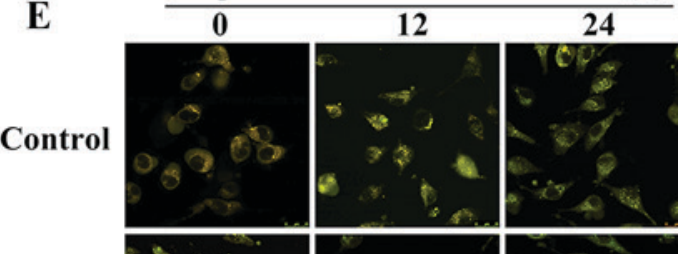

HO-1
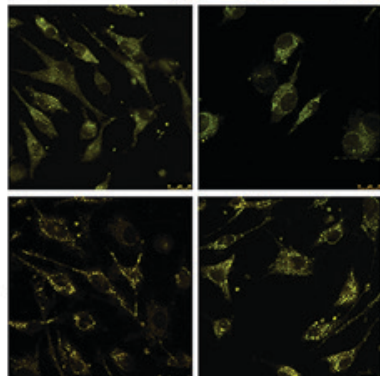

B

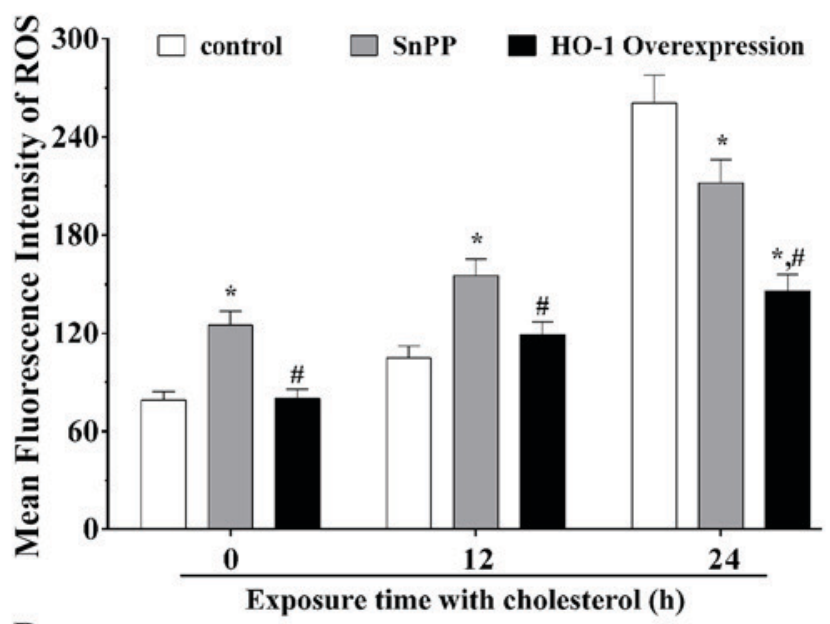

D
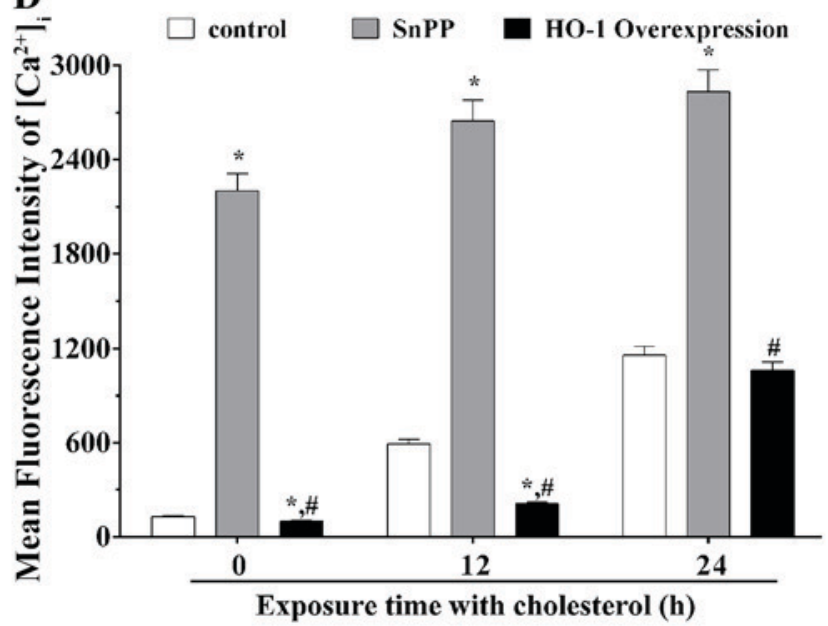

F

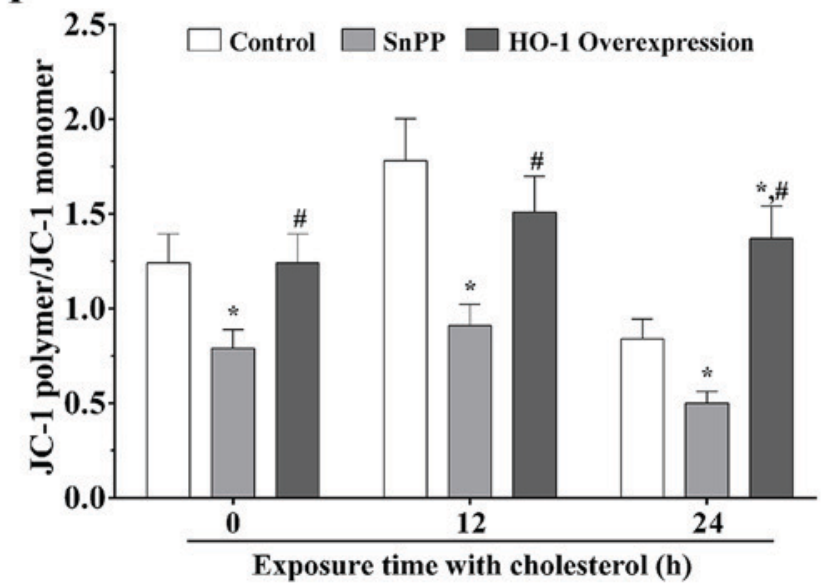

Figure 4. Measurements of $\Delta \Psi \mathrm{m},\left[\mathrm{Ca}^{2+}\right]_{\mathrm{i}}$ and $[\mathrm{ROS}]_{\mathrm{i}}$ in EA.hy926 cells stimulated with cholesterol. (A) Changes in the concentrations of [ROS $]_{\mathrm{i}}$ in EA.hy926 cells following stimulation with cholesterol using confocal microscopy (magnification, x200). (B) The quantification results of mean fluorescence intensity of $[R O S]_{i}$ in EA.hy926 cells following stimulation with cholesterol. (C) Changes in the concentrations of $\left[\mathrm{Ca}^{2+}\right]_{i}$ in EA.hy926 cells following stimulation with cholesterol using confocal microscopy (magnification, x200). (D) The quantification results of mean fluorescence intensity of $\left[\mathrm{Ca}^{2+}\right]_{\mathrm{i}}$ in EA.hy926 cells following stimulation with cholesterol. (E) Changes of $\Delta \Psi \mathrm{m}$ in EA.hy926 cells following stimulation with cholesterol using confocal microscopy (magnification, $\mathrm{x} 200$ ). (F) Ratio of JC-1 polymer/JC-1 monomer in EA.hy926 cells following stimulation with cholesterol. "P<0.05 vs. control group; ${ }^{\sharp} \mathrm{P}<0.05$ vs. SnPP group. HO-1, heme oxygenase 1; SnPP, tin protoporphyrin; $\Delta \Psi \mathrm{m}$, mitochondrial membrane potential; $\left[\mathrm{Ca}^{2+}\right]_{\mathrm{i}}$, intercellular $\mathrm{Ca}^{2+}$; $\mathrm{ROS}$, reactive oxygen species.

glucose and viral infection, in which it exhibits cytoprotective effects $(18,19)$. The results of the present study indicated that the expression of HO-1 in EA.hy926 cells following stimulation with cholesterol for $12 \mathrm{~h}$ was increased. However, this expression decreased following stimulation with cholesterol for $24 \mathrm{~h}$. These results suggested that EA.hy 926 cells gradually restored to a normal physiological status. The overexpression HO-1 at the transcriptional level is essential 
to adapt to cholesterol-mediated oxidative stress. Previous studies have confirmed that HO-1 can be activated by hypoxia, and the present study showed that the expression of HO-1 was increased with alleviation of the cell injury caused by a combination of cholesterol stimulation and SnPP inhibition in the EA.hy926 cells (18).

Several signal transduction pathways can induce HO-1 expression via the activation of different transcription factors, including BTB domain and CNC homolog 2, P53, cAMP response element binding protein and $\mathrm{Nrf} 2$ (20). EA.hy926 cells have different defense systems in response to oxidative stress, including phase I enzymes and phase II enzymes (21). The results of the present study showed that the expression levels of Nrf2 in EA.hy926 cells stimulated with cholesterol were increased, and the activation of Nrf2 promoted the upregulation in the expression of HO-1. Under oxidative stress, Nrf2 is released from Keap1 and translocated to the nucleus, where it binds to AREs in the promoter/enhancer region of antioxidant enzyme genes of the phase II anti-oxidative system, including HO-1. Nrf2 can upregulate the expression of these antioxidant enzymes, and has a cytoprotective role $(22,23)$. The results of the present study also showed that the $\left[\mathrm{Ca}^{2+}\right]$ i was increased following stimulation with cholesterol, and the concentration of $\left[\mathrm{Ca}^{2+}\right]_{\mathrm{i}}$ in the HO-1-overexpressing group was significantly decreased, indicating that the expression of $\mathrm{HO}-1$ may be a negative regulator of $\left[\mathrm{Ca}^{2+}\right]_{i} \cdot\left[\mathrm{Ca}^{2+}\right]_{i}$, as a second messenger in several signaling pathways, promotes the translocation of Nrf2 from the cytoplasm to the nucleus, and functions as a transcriptional factor to regulate downstream molecules (24). The activation of Nrf2 signaling increases the expression of $\mathrm{HO}-1$, activates the phase II antioxidant response, and exerts its anti-oxidative function to protect endothelial cells.

$N F-\kappa B$ consists of a family of transcription factors, including p52, p50, c-rel, RelA (p65) and RelB, and is a key transcription factor, which mediates immune responses to inflammation and cell proliferation, and protects against UV radiation (25). Several studies have shown that the generation of ROS can subsequently activate NF- $\kappa \mathrm{B}(26,27)$. The results of the present study showed that expression levels of the $\mathrm{NF}-\kappa \mathrm{B}$ p65 subunit were increased following exposure to cholesterol, indicating that cholesterol induced the accumulation of ROS in EA.hy926 cells (28) and then caused the upregulation of $N F-\kappa B$. Studies have also shown that the p65 subunit of $\mathrm{NF}-\kappa \mathrm{B}$ assists in increasing the level of nuclear Keap1. Keap1 can bind with Nrf2, decreasing the binding of Nrf2 with AREs in the promoter/enhancer region, and then inhibits the activation of antioxidant enzyme genes in the phase II anti-oxidative system, including the expression of HO-1 (29,30). In addition, p65 can promote the interaction of histone deacetylase 3 with MAF bZIP transcription factor $\mathrm{K}$ (MafK), preventing the heterodimer formation of MafK with Nrf2 and decreasing the expression of ARE-associated genes (31).

The MAPK signaling pathways, including ERK1/2, p38 and c-Jun N-terminal kinase, are the regulators of the expression of HO-1 under extracellular stimulation. MAPKs act as positive and negative regulators between the extracellular stimulation and expression of HO-1, depending on different cell types and various extracellular stimulations (32). The results of the present study showed that the ratios of
p-ERK/ERK were increased in the three groups following stimulation with cholesterol. MAPKs, as critical signaling pathways, are involved in the activation and translocation of Nrf2 for the synthesis of essential proteins, particularly HO-1. ERK signaling can promote the Nrf2 phosphorylation process, which can induce the release of Nrf2 from the Keap1-Nrf2 complex and cause the translocation of Nrf2 from the cytoplasm into the nucleus, inducing the upregulation of HO-1. ERK is involved in cellular responses under the stimulation of a number of growth and differentiation factors; however, several studies have confirmed that $\mathrm{HO}-1$ is also induced by the ERK signaling pathway under physiological conditions $(23,33)$. According to the results of the present study, the ERK signaling pathway was activated under stimulation with cholesterol, which resulted in the upregulated expression of HO-1 and a cytoprotective effect in EA.hy926 cells.

The present study showed that the expression levels of $\mathrm{NF}-\kappa \mathrm{B}$ in the three groups were upregulated following stimulation with cholesterol for $12 \mathrm{~h}$, and were decreased following stimulation for $24 \mathrm{~h}$. The ratio of $\mathrm{p}-\mathrm{ERK} / \mathrm{ERK}$ was increased following stimulation with cholesterol for 12 and $24 \mathrm{~h}$ in the control and SnPP treatment groups. The ratio in the HO-1 overexpression group was increased following stimulation with cholesterol for $12 \mathrm{~h}$. Therefore, it was hypothesized that endothelial cells can alleviate cholesterol-induced oxidative stress via the activation and translocation of Nrf2, activation of the MAPK/ERK signaling pathway and increased concentration of $\left[\mathrm{Ca}^{2+}\right]_{\mathrm{i}}$ to promote the expression of $\mathrm{HO}-1$ in EA.hy926 cells.

Several studies have shown that oxidative stress can decrease the expression of several survival signaling molecules, including PI3K, p-AKT and $\mathrm{Bcl}-2$. PI3K pathway activation is involved in cell proliferation and cell survival in response to cytokines and growth factors (34-36). In the present study, it was found that the ratio of $\mathrm{p}$-AKT/AKT was decreased following stimulation with cholesterol, indicating that the PI3K/AKT signaling pathway was inhibited by stimulation of cholesterol. p-AKT can inhibit several cytosolic pro-apoptotic substrates. The results of the present study suggested that cholesterol increased ROS concentrations, which may significantly increase the expression of HO-1. The ratio of p-AKT/AKT deceased as the expression of HO-1 increased, therefore, the overexpression of HO-1 decreased the ratio of p-AKT/AKT in endothelial cells under cholesterol stimulation. These results suggested that the upregulation of HO-1 significantly inhibited the cholesterol-activated PI3K/AKT signaling pathway.

The present study also found that expression levels of c-Myc were decreased under stimulation with cholesterol. Upregulation of the expression of HO-1 may indirectly suppress the expression level of c-Myc. Studies have shown that the overexpression of c-Myc leads to a marked reduction in the phosphorylation of enhancer of zeste homolog 2 (EZH2) in phoenix cells and immortalizes mammary epithelial cells (37). c-Myc can phosphorylate AKT at the S21 site and suppress the activation of AKT in parallel with reduced phosphorylation of EZH2. Similarly, the apoptotic effect or mechanisms of c-Myc are primarily involved in the regulation of BCL family members at the transcription stage, including pro-apoptotic $\mathrm{Bcl}-2$-associated $\mathrm{X}$ protein, $\mathrm{Bcl}-2$ 
antagonist killer and p53 upregulated modulator of apoptosis/Bcl-2-binding component 3. Studies have shown that the potent apoptotic potential of c-Myc can also be enforced via the $\mathrm{p} 14 \mathrm{ARF} / \mathrm{p} 53$ axis $(38,39)$.

Therefore, the results of the present study suggested that cholesterol induced the oxidative stress status and increased the generation of ROS in endothelial cells. This accumulation of ROS subsequently resulted in the upregulated expression of HO-1. The present study found that cholesterol stimulation increased the expression of Nrf2 and the concentration of $\left[\mathrm{Ca}^{2+}\right]_{i}$, activating ERK signaling and inducing the overexpression of HO-1. HO-1 exerted a cytoprotective effect through the inhibition of PI3K/AKT signaling and by decreasing the expression of c-Myc in EA.hy926 cells.

\section{Acknowledgements}

This study was supported by grants from the National Natural Science Foundation of China (grant no. 81570335), the Tianjin Natural Science Foundation (grant no. 15ZXJZSY00010 and 12JCYBJC15900) and the Opening Funding for Tianjin Key Laboratory of Cardiovascular Remodeling and Target Organ Injury (grant no. TJC1401).

\section{References}

1. Morgan AE, Mooney KM, Wilkinson SJ, Pickles NA and Mc Auley MT: Cholesterol metabolism: A review of how ageing disrupts the biological mechanisms responsible for its regulation. Ageing Res Rev 27: 108-124, 2016.

2. Janoudi A, Shamoun FE, Kalavakunta JK and Abela GS Cholesterol crystal induced arterial inflammation and destabilization of atherosclerotic plaque. Eur Heart J 37: 1959-1967, 2016

3. Korytowski W, Wawak K, Pabisz P, Schmitt JC, Chadwick AC, Sahoo D and Girotti AW: Impairment of macrophage cholesterol efflux by cholesterol hydroperoxide trafficking: Implications for atherogenesis under oxidative stress. Arterioscler Thromb Vasc Biol 35: 2104-2113, 2015.

4. Zhang J, Wang X, Vikash V, Ye Q, Wu D, Liu Y and Dong W: ROS and ROS-mediated cellular signaling. Oxid Med Cell Longev 2016: 4350965, 2016.

5. Martínez-Reyes I and Cuezva JM: The H(+)-ATP synthase: A gate to ROS-mediated cell death or cell survival. Biochim Biophys Acta 1837: 1099-1112, 2014.

6. Fang J, Seki T and Maeda H: Therapeutic strategies by modulating oxygen stress in cancer and inflammation. Adv Drug Deliv Rev 61: 290-302, 2009.

7. Cao J, Inoue K, Sodhi K, Puri N, Peterson SJ, Rezzani R and Abraham NG: High-fat diet exacerbates renal dysfunction in SHR: Reversal by induction of HO-1-adiponectin axis. Obesity (Silver Spring) 20: 945-953, 2012

8. Liemburg-Apers DC, Willems PH, Koopman WJ and Grefte S: Interactions between mitochondrial reactive oxygen species and cellular glucose metabolism. Arch Toxicol 89: 1209-1226, 2015.

9. Andrisse S, Koehler RM, Chen JE, Patel GD, Vallurupalli VR, Ratliff BA, Warren DE and Fisher JS: Role of GLUT1 in regulation of reactive oxygen species. Redox Biol 2: 764-771, 2014.

10. Lv X, Song DM, Niu YH and Wang BS: Inhibition of heme oxygenase-1 enhances the chemosensitivity of laryngeal squamous cell cancer Hep-2 cells to cisplatin. Apoptosis 21: 489-501, 2016.

11. Gozzelino R, Jeney V and Soares MP: Mechanisms of cell protection by heme oxygenase-1. Annu Rev Pharmacol Toxicol 50: 323-354, 2010.

12. Song G, Zong C, Zhang Z, Yu Y, Yao S, Jiao P, Tian H, Zhai L, Zhao H, Tian S, et al: Molecular hydrogen stabilizes atherosclerotic plaque in low-density lipoprotein receptor-knockout mice. Free Radic Biol Med 87: 58-68, 2015.

13. Wu BJ, Chen K, Shrestha S, Ong KL, Barter PJ and Rye KA: High-density lipoproteins inhibit vascular endothelial inflammation by increasing $3 \beta$-hydroxysteroid- $\Delta 24$ reductase expression and inducing heme oxygenase-1. Circ Res 112: 278-288, 2013.
14. O'Reilly S, Ciechomska M, Cant R and van Laar JM: Interleukin-6 (IL-6) trans signaling drives a STAT3-dependent pathway that leads to hyperactive transforming growth factor- $\beta$ (TGF- $\beta$ ) signaling promoting SMAD3 activation and fibrosis via Gremlin protein. J Biol Chem 289: 9952-9960, 2014.

15. Li X, Ren Y, Sorokin V, Poh KK, Ho HH, Lee CN, de Kleijn D, Lim SK, Tam JP and Sze SK: Quantitative profiling of the rat heart myoblast secretome reveals differential responses to hypoxia and re-oxygenation stress. J Proteomics 98: 138-149, 2014.

16. Li J, Song J, Bi S, Zhou S, Cui J, Liu J and Wu D: Electrochemical estrogen screen method based on the electrochemical behavior of MCF-7 cells. J Hazard Mater 313: 238-243, 2016.

17. Liu F, Tang W, Chen D, Li M, Gao Y, Zheng H and Chen S: Expression of TGF- $\beta 1$ and CTGF is associated with fibrosis of denervated sternocleidomastoid muscles in mice. Tohoku J Exp Med 238: 49-56, 2016.

18. Abraham NG and Kappas A: Pharmacological and clinical aspects of heme oxygenase. Pharmacol Rev 60: 79-127, 2008

19. Li X, Ye F, Li L, Chang W, Wu X and Chen J: The role of HO-1 in protection against lead-induced neurotoxicity. Neurotoxicology 52: 1-11, 2016.

20. Ning W, Song R, Li C, Park E, Mohsenin A, Choi AM and Choi ME: TGF-beta1 stimulates HO-1 via the p38 mitogen-activated protein kinase in A549 pulmonary epithelial cells. Am J Physiol Lung Cell Mol Physiol 283: L1094-L1102, 2002.

21. Buendia I, Michalska P, Navarro E, Gameiro I, Egea J and León R: Nrf2-ARE pathway: An emerging target against oxidative stress and neuroinflammation in neurodegenerative diseases. Pharmacol Ther 157: 84-104, 2016.

22. Chen B, Lu Y, Chen $Y$ and Cheng J: The role of Nrf2 in oxidative stress-induced endothelial injuries. J Endocrinol 225: R83-R99, 2015.

23. Na HK and Surh YJ: Oncogenic potential of Nrf2 and its principal target protein heme oxygenase-1. Free Radic Biol Med 67: 353-365, 2014.

24. Santofimia-Castaño P, Clea Ruy D, Garcia-Sanchez L, Jimenez-Blasco D, Fernandez-Bermejo M, Bolaños JP, Salido GM and Gonzalez A: Melatonin induces the expression of Nrf2-regulated antioxidant enzymes via PKC and $\mathrm{Ca} 2+$ influx activation in mouse pancreatic acinar cells. Free Radic Biol Med 87: 226-236, 2015.

25. Liu Y, Zhou G, Wang Z, Guo X, Xu Q, Huang Q and Su L: NF-кB signaling is essential for resistance to heat stress-induced early stage apoptosis in human umbilical vein endothelial cells. Sci Rep 5: 13547, 2015.

26. Xu B, Wang S, Li R, Chen K, He L, Deng M, Kannappan V, Zha J, Dong $\mathrm{H}$ and Wang W: Disulfiram/copper selectively eradicates AML leukemia stem cells in vitro and in vivo by simultaneous induction of ROS-JNK and inhibition of NF- $\kappa \mathrm{B}$ and Nrf2. Cell Death Dis 8: e2797, 2017.

27. Chao W, Deng JS, Li PY, Liang YC and Huang GJ: 3,4-Dihydroxybenzalactone suppresses human non-smallcelllung carcinoma cells metastasis via suppression of epithelial to mesenchymal transition, ROS-Mediated PI3K/AKT/MAPK/MMP and NFкB signaling pathways. Molecules 22: pii: E537, 2017.

28. Tornatore L, Thotakura AK, Bennett J, Moretti M and Franzoso G: The nuclear factor kappa B signaling pathway: Integrating metabolism with inflammation. Trends Cell Biol 22: 557-566, 2012.

29. Hoetzel A, Vagts DA, Loop T, Humar M, Bauer M, Pahl HL, Geiger KK and Pannen BH: Effect of nitric oxide on shock-induced hepatic heme oxygenase-1 expression in the rat. Hepatology 33: 925-937, 2001.

30. Huang CS, Lin AH, Yang TC, Liu KL, Chen HW and Lii CK: Shikonin inhibits oxidized LDL-induced monocyte adhesion by suppressing $\mathrm{NF} \kappa \mathrm{B}$ activation via up-regulation of PI3K/Akt/Nrf2-dependent antioxidation in EA.hy926 endothelial cells. Biochem Pharmacol 93: 352-361, 2015.

31. Wardyn JD, Ponsford AH and Sanderson CM: Dissecting molecular cross-talk between Nrf2 and NF- $\kappa \mathrm{B}$ response pathways. Biochem Soc Trans 43: 621-626, 2015.

32. Wu BJ, Chen K, Barter PJ and Rye KA: Niacin inhibits vascular inflammation via the induction of heme oxygenase-1. Circulation 125: 150-158, 2012.

33. Cai C, Teng L, Vu D, He JQ, Guo Y, Li Q, Tang XL, Rokosh G, Bhatnagar A and Bolli R: The heme oxygenase 1 inducer (CoPP) protects human cardiac stem cells against apoptosis through activation of the extracellular signal-regulated kinase (ERK)/NRF2 signaling pathway and cytokine release. J Biol Chem 287: $33720-33732,2012$. 
34. Ying C, Chen L, Wang S, Mao Y, Ling H, Li W and Zhou X Zeaxanthin ameliorates high glucose-induced mesangial cell apoptosis through inhibiting oxidative stress via activating AKT signalling-pathway. Biomed Pharmacother 90: 796-805, 2017.

35. Peng HB, Wang RX, Deng HJ, Wang YH, Tang JD, Cao FY and Wang JH: Protective effects of oleanolic acid on oxidative stress and the expression of cytokines and collagen by the AKT/NF- $\kappa B$ pathway in silicotic rats. Mol Med Rep 15: 3121-3128, 2017.

36. Tang R, Xu X, Yang W, Yu W, Hou S, Xuan Y, Tang Z, Zhao S, Chen Y, Xiao X, et al: MED27 promotes melanoma growth by targeting AKT/MAPK and NF- $\kappa \mathrm{B} / \mathrm{iNOS}$ signaling pathways. Cancer Lett 373: 77-87, 2016.
37. Kaur M and Cole MD: MYC acts via the PTEN tumor suppressor to elicit autoregulation and genome-wide gene repression by activation of the Ezh2 methyltransferase. Cancer Res 73: 695-705, 2013.

38. Calvisi DF, Ladu S, Hironaka K, Factor VM and Thorgeirsson SS: Vitamin E down-modulates iNOS and NADPH oxidase in c-Myc/TGF-alpha transgenic mouse model of liver cancer. J Hepatol 41: 815-822, 2004.

39. McMahon SB: MYC and the control of apoptosis. Cold Spring Harb Perspect Med 4: a014407, 2014. 